\title{
Increased Brain Levels of F2-Isoprostane Are an Early Marker of Behavioral Sequels in a Rat Model of Global Perinatal Asphyxia
}

\author{
GEMMA CALAMANDREI, ALDINA P. VENEROSI, ANGELA VALANZANO, \\ MARIA ANNA DE BERARDINIS, ANITA GRECO, MARIA PUOPOLO, AND LUISA MINGHETTI \\ Section of Comparative Psychology [G.C., A.P.V., A.V., M.P.] and Section of Neurobiology [M.A.D.B., \\ A.G., L.M.], Laboratorio di Fisiopatologia O.S., Istituto Superiore di Sanità, Viale Regina Elena 299, \\ I-00161 Roma, Italy
}

\begin{abstract}
Perinatal asphyxia is a major cause of immediate and postponed brain damage in the newborn. It may be responsible for several delayed neurologic disorders and, in this respect, early markers of brain injury would be relevant for therapeutic intervention as well as for identification of infants at high risk for developmental disabilities. Biochemical measurements (brain $\mathrm{F}_{2}$-isoprostane levels) and behavioral tests (ultrasonic vocalization pattern on postnatal days (pnd) 5, 8, and 11, spontaneous motor behaviors on pnd 7 and 12, and homing response on pnd 10) were performed in a rat model of global perinatal asphyxia in the immature neonate. Caesarean section was performed in rats and the pups, still in uterus horns, were placed into a water bath at $37^{\circ} \mathrm{C}$ for either 10 or $20 \mathrm{~min}$. Caesarean delivered pups were used as controls. Pups experiencing severe (20 $\mathrm{min}$ ), in contrast to those undergoing the $10 \mathrm{~min}$, asphyctic insult presented with detectable abnormalities including early (two hours after the insult) increase in brain $\mathrm{F}_{2}$-isoprostane (a direct marker of oxidative injury) without detectable changes in $\mathrm{PGE}_{2}, \mathrm{COX}-2$ and iNOS levels, and delayed physical (reduced weight gain on pnd 5 and thereafter) and behavioral disturbances (alterations in ultrasound emission on pnd 11 and spontaneous motricity levels mainly). These findings suggest that increased brain $\mathrm{F}_{2}$-isoprostane levels shortly after the asphyctic insult are predictive of delayed behavioral disturbances in the newborn rat. The present 20-min asphyxia model might serve for the assessment of preventive and curative strategies
\end{abstract}

\section{ABSTRACT}

to treat neurologic/behavioral disturbances associated with perinatal asphyxia. (Pediatr Res 55: 85-92, 2004)
CNS, central nervous system
EEG, electroencephalography
MRI, magnetic resonance imaging
CSF, cerebrospinal fluid
VLBW, very low birth weight
VD, vaginally delivered
BHT, butylated hydroxytoluene
COX, cyclooxygenase
PGE $_{2}$, prostaglandin $\mathrm{E}_{2}$
PMSF, phenylmethanesulfonyl fluoride
EDTA, ethylene diamine tetraacetic-acid

EGTA, ethylene glycol-bis (2-aminoetylether)-N,N,N',N'-

tetraacetic-acid

SDS-PAGE, SDS-polyacrylamide gel electrophoresis

INOS, inducible nitric oxide synthase

ECL, enhanced chemiluminescence

ANOVA, analysis of variance

Pnd, postnatal day

ANCOVA, analysis of covariance
Perinatal asphyxia is a major cause of neonatal mortality and irreversible damage to the brain. Severe asphyxia may induce major deficits (cerebral palsy, dystonia, epilepsy) shortly after birth (1), while mild/moderate asphyxia episodes may result in

Received November 12, 2002; accepted May 9, 2003.

Correspondence: Gemma Calamandrei, Section of Comparative Psychology, Laboratorio di Fisiopatologia, Istituto Superiore di Sanità, Viale Regina Elena 299, I-00161 Roma, Italy; e-mail: gemma.calamandrei@iss.it

Supported by a grant from the Italian Ministry of Health, Project No. 0AN/F "Hypoxic/ ischemic brain damage in the newborn: epidemiological and experimental studies on diagnosis, therapies and rehabilitation" subprojects No. 0AN/F7 and 0AN/F9.

DOI: 10.1203/01.PDR.0000099774.17723.D4 cognitive/attentional disorders later on in development. A critical concept emerging from recent research is that of a "therapeutic window." namely a narrow period of time between the insult and the occurrence of CNS injury, during which adoption of neuroprotective strategies could be effective $(2,3)$. A primary aim for clinical research is to identify as early as possible reliable indexes of brain injury in the asphyctic newborns to apply potential therapeutic interventions at the optimal time and to identify those infants at high risk for developmental delays and disabilities (4).

To date the major advances in the early prediction of hypoxic-ischemic insult have been attained by means of EEG and 
evoked potentials (5) and the more recent neuroimaging methodologies such as MRI $(6,7)$ and MR spectroscopy $(8)$. However, the predictive value of these techniques in preterms is limited. Neurologic examination of the newborn may also provide unique information for an early diagnosis of neurologic disorders (9). In particular, a new approach to clinical neurologic evaluation of both term and preterm newborns was introduced by Prechtl (10), based on qualitative observation of spontaneous motility, which resulted highly correlated with both presence of brain lesion and neurologic outcome (11). In addition to these approaches, the identification of potential biochemical markers - originated within the brain and released into CSF, blood, or urine after the hypoxic-ischemic insult might ultimately represent relevant prognostic and diagnostic tools for assessing occurrence and severity of brain injury in the newborn. In this line, two recent studies reported changes in the serum profile of three brain proteins after birth asphyxia (12) and increased CSF levels of $\mathrm{F}_{2}$-isoprostanes, an index of lipid peroxidation, in VLBW infants with white matter injury (13).

In this study we used a rat model of global perinatal asphyxia that mimics an acute intrauterine asphyxia event around the time of birth (14). Due to the different degree of CNS maturity at birth between rodents and humans (15), this asphyxia model relates more to the condition of the premature rather than the term human infant (16). In this model, neural and behavioral alterations have been identified (17-20). Shortterm effects ( $8 \mathrm{~d}$ after induction of asphyxia) involve delayed cell death in frontal cortex, striatum, and cerebellum $(20,21)$ and increased dopamine levels in mesencephalic and telencephalic regions (19). Long-term changes involve alterations in dopaminergic system (22) and in myelination patterns (23), as well as motor alterations and slight impairments in retention of a spatial learning task (17). The aim of our investigation was to assess the occurrence of biochemical alterations in the brain of asphyctic rat pups shortly after the insult, which could be related to abnormalities in neonatal behavior patterns, with respect to ultrasonic vocalization pattern, spontaneous motor behaviors, and homing response. Given the potential role of oxidative stress in hypoxic injury (24), and the recent findings in human infants (13), we chose to analyze changes in brain level of $\mathrm{F}_{2}$-isoprostanes.

These prostaglandin-like compounds are formed by free radical-catalyzed peroxidation of arachidonic acid within cell membranes and they are reliable markers of lipid peroxidation and oxidative stress in vivo (25). Since their discovery, $\mathrm{F}_{2}-$ isoprostanes, and in particular the 8-epi- $\mathrm{PGF}_{2 \alpha}$, a major $\mathrm{F}_{2}-$ isoprostanes formed in vivo, have been successfully used to investigate the role of oxidative stress in neurodegenerative and demyelinating diseases (26 and references therein).

\section{MATERIAL AND METHODS}

Animals and breeding procedures. Wistar rats were purchased from Charles River Italia (Calco, Italy) and kept in air-conditioned room at $21 \pm 1{ }^{\circ} \mathrm{C}$ and $60 \pm 10 \%$ relative humidity, with a white red light cycle (white light on from 8.30 to 20.30). One week after the arrival of the rats, breeding pairs were formed, and after $48 \mathrm{~h}$, females were individually housed until the 22nd day of gestation, when the caesarean section was carried out.

Experimental procedures. The experimental protocol was conducted according to the EC guidelines (EC Council Directive 86/609 1987) and the Italian legislation, and under permission of the Italian Ministry of Health. Asphyxia was induced in pups delivered by caesarean section on pregnant Wistar rats, as described by (14). Time-mated female rats within the last day of gestation were decapitated and the entire uterus was quickly removed. Use of anesthetic in the dam was avoided because maternal anesthesia affects the degree of CNS hypoxia experienced by the neonate. Immediately after hysterectomy, four pups were delivered to be considered caesareandelivered (0-min asphyxia) controls, then the uterus horns, still containing the fetuses were placed in a saline bath at $37^{\circ} \mathrm{C}$. Pups from the same uterus were then removed after 10, or 20 min and stimulated to breath by gently touching them around the oral and genital areas for approximately $10 \mathrm{~min}$. Fetuses were assigned randomly to the different asphyxia condition. Pups were then left to recover for approximately $1 \mathrm{~h}$ in a humidity- and temperature-controlled incubator at $30 \pm 1{ }^{\circ} \mathrm{C}$ (Elmed Ginevri 0GB 1000, Rome, Italy) until fostered by surrogate mothers that had given birth to healthy litters within $24 \mathrm{~h}$. One male and one female pup were removed within $12 \mathrm{~h}$ after birth from surrogate mothers to obtain a VD (vaginal delivered) control group (to assess potential effects of the caesarean section on the behavioral parameters examined) and compared with the 0-min asphyxia controls. Litters were built assigning all the pups coming from the same donor mother to a foster dam, to ensure that fetuses from a donor mother contributed equally to both behavioral and biochemical analysis. One male and one female from each asphyxia condition (0-min, 10-min, 20-min) were assigned to a foster dam, together with one male and one female of VD group (8 pups/ litter, for a total of 10 litters). All the pups from these ten litters were used for assessment of motor behavior and ultrasonic vocalization. As for the remaining pups from the same donor mother, at least one pup (one male and one female whenever possible) in each asphyxia condition (0-min, 10-min, 20-min), was sacrificed for biochemical studies. The random procedure used in assigning pups from the same donor mother to behavioral or biochemical tests excluded any possible bias in building the two experimental sets of animals. Pups used for the homing test derived from a previous experiment in which only male pups were retained within the litter ( $n=12$ litters).

Preparation of brain homogenates and assessment of biochemical alterations. Pups assigned to biochemical analysis (0-min, $n=17$, 9F, 8M; 10-min, $n=17$, 8F, 9M; 20-min, $n=$ $13,7 \mathrm{~F}, 6 \mathrm{M}$ ) were decapitated $2 \mathrm{~h}$ after birth and brains rapidly dissected out, snap frozen in liquid $\mathrm{N}_{2}$ and stored at $-80^{\circ} \mathrm{C}$ until required. Tissue samples were extracted as previously described (24). Briefly, a single hemisphere from each brain was weighed (50-100 $\mathrm{mg}$ of tissue) and homogenized in 0.5 $\mathrm{mL}$ of $50 \mathrm{mM}$ Tris buffer, $\mathrm{pH} 7.5$ containing $10 \mu \mathrm{g} / \mathrm{mL}$ indomethacin and $10 \mu \mathrm{M}$ BHT to block COX activity and spontaneous oxidation, respectively. Homogenates were vigorously vortexed and incubated for $5 \mathrm{~min}$ on ice before cen- 
trifuging at $14,000 \mathrm{rpm}$ for $45 \mathrm{~min}$. Supernatants were collected and stored at $-80^{\circ} \mathrm{C}$ until required. With this procedure, only the $\mathrm{F}_{2}$-isoprostanes and not the membrane-bound fraction, was extracted for a better representation of the metabolite, which is likely to be naturally released at early times after asphyxia. Undiluted samples were then tested for $\mathrm{F}_{2}$ isoprostane and $\mathrm{PGE}_{2}$ content.

The $\mathrm{F}_{2}$-isoprostane 8-epi-PGF $\mathrm{PG}_{2 \alpha}$ was measured by a colorimetric enzyme immunoassay (detection limit: $2 \mathrm{pg} / \mathrm{mL}$; Cayman Chemical, Ann Arbor, MI, U.S.A.), according to the manufacturer instructions. The sensitivity of 8 -epi- $\mathrm{PGF}_{2 \alpha}$ assay was improved from 4 to $2 \mathrm{pg} / \mathrm{mL}$, by optimizing the incubation time of the developing reaction. $\mathrm{PGE}_{2}$ was measured by a chemiluminescence enzyme immunoassay (detection limit: $1.6 \mathrm{pg} / \mathrm{mL}$; Assay Design, Inc., Ann Arbor, MI, U.S.A.). All measurements were done at least in duplicate.

For Western blot analysis tissue samples were homogenized in $50 \mathrm{mM}$ Tris buffer, $\mathrm{pH} 7.5$ supplemented with $1 \% \mathrm{NP} 40$, $0.1 \% \mathrm{SDS}, 100 \mu \mathrm{g} / \mathrm{mL}$ PMSF, $30 \mu \mathrm{g} / \mathrm{mL}$ aprotinin, $100 \mu \mathrm{M}$ leupeptin, $10 \mathrm{mM} \mathrm{NaF}, 1 \mathrm{mM}$ EDTA, $1 \mathrm{mM}$ EGTA, $100 \mu \mathrm{M}$ $\left.\mathrm{Na}_{3} \mathrm{VO}_{4}\right)$ and insoluble material removed by centrifugation $\left(10,000 \times g\right.$ at $\left.4^{\circ} \mathrm{C}, 10 \mathrm{~min}\right)$. Brain tissue from cortex and hippocampus of adult rats as positive controls were prepared with the same procedure. Equals amounts of proteins (50 $\mu \mathrm{g} / \mathrm{lane})$ were separated by 10 or $12 \%$ SDS-PAGE and transferred to nitrocellulose membranes, then blocked with $10 \%$ nonfat milk and incubated with polyclonal anti-iNOS $(1: 2500)$ or anti-COX-2 (1:500) antibodies (Cayman Chemical, Ann Arbor, MI, U.S.A.) for $1 \mathrm{~h}$ at $25^{\circ} \mathrm{C}$. Horseradish peroxidase conjugated anti-rabbit $\operatorname{IgG}\left(1: 5000,1 \mathrm{~h}\right.$ at $\left.25^{\circ} \mathrm{C}\right)$ and $\mathrm{ECL}$ reagents from Amersham (Buckingamshire, UK) were used as detection system. Purified COX-2 and iNOS were used as standard controls $(0.5 \mu \mathrm{g} / \mathrm{lane})$.

Spontaneous behavior (pnds 7, 12). One male and one female pup of each experimental condition from each litter (VD, $n=10 \mathrm{~F}, 10 \mathrm{M}$; 0-min, $n=10 \mathrm{~F}, 10 \mathrm{M}$; 10-min, $n=10$ $\mathrm{F}, 10 \mathrm{M}$; 20-min, $n=10 \mathrm{~F}, 10 \mathrm{M}$ ) were individually introduced in a glass cylinder (14 cm diameter, $8 \mathrm{~cm}$ height) with adsorbent paper on the floor kept in an incubator set at $30 \pm 1{ }^{\circ} \mathrm{C}$. Pups' behavior was then video-recorded for $15 \mathrm{~min}$. Frequency and duration of the following behavioral items were collected (27): Locomotion (forward movements of the body); Laying still (no visible movements of the body); Head moving (head raising and head turning); Wall climbing; Twitching (a definite abrupt twitch of the body, considered a behavioral index of "active sleep"); Face washing (signs of wiping); Rolling (roll of the body).

Ultrasonic vocalization (pnds 5, 8, and 11). Ultrasound recording was carried out as described in (28). One male and one female pup of each experimental condition (VD, $n=9 \mathrm{~F}$, $9 \mathrm{M}$; 0-min, $n=9$ F, 9 M; 10-min, $n=9$ F, 9 M; 20-min, $n$ $=9 \mathrm{~F}, 9 \mathrm{M}$; one litter was excluded from this test for technical reasons) were removed from each litter and single pup's ultrasound were recorded aurally for $3 \mathrm{~min}$ in a soundattenuating chamber maintained at ambient temperature (28 \pm $1{ }^{\circ} \mathrm{C}$ ), by using a Bat Detector (QMC Instruments, London, E1 4AA, UK, Model S200, frequency range: $10-180 \mathrm{kHz}$ ).
Homing test (pnd 10). Homing test was carried out as described in (29). Briefly, within each litter, male pups assigned to the different experimental conditions (VD, $n=12$; 0-min, $n=7$; 10-min, $n=7$; 20-min, $n=10$ ) were assessed for their homing response in a Plexiglas arena $(36 \times 22.5 \times 10$ $\mathrm{cm}$ ) maintained at $28 \pm 1{ }^{\circ} \mathrm{C}$, whose floor was subdivided into 15 quadrants. Wood shavings from the home cage were evenly spread under the wire-mesh floor on one side of the arena and the pup was placed on the opposite side. The time required for each pup to place both forelimbs within the goal area and the number of quadrants entered was recorded (cut-off time $3 \mathrm{~min}$ ).

Statistical analysis. Statistical analysis was carried out by means of BMDP software package (version BMDP/dynamic 7.0, Berkeley, CA, U.S.A.). ANOVAs were performed on parametric data concerning pups' body weight, ultrasound vocalization, and spontaneous behavior. The models of analysis included litter as random factor and littermates as repeated measures of litter (30). Post hoc comparisons after parametric results were performed by Tukey's test (31). Biochemical data were analyzed by parametric methods due to symmetrical distribution and absence of values out of the detection limit. Nonparametric tests were used for homing data.

Differences between VD and 0-min asphyxia controls were evaluated. Data are reported by means and SEM. When multiple interactions are represented, pooled SEM value was chosen.

\section{RESULTS}

No significant differences were found between VD and caesarean controls in any of the test performed. Therefore, in the Result section, and relative figures, only results obtained on 0-min, 10-min, and 20-min asphyxia groups were reported.

Mortality rate and body weight. Survival was $95 \%$ following $20 \mathrm{~min}$ of birth asphyxia and $100 \%$ in the 0 - and 10 -min asphyxia groups. These survival rates were significantly higher than those reported by other authors using the same global asphyxia model, most likely because of differences in the experimental procedure. At variance with previous studies, we performed caesarean delivery about six hours before birth, and not after delivery of the first pup. Furthermore, after resuscitation procedure, pups were left to recover in a temperature and humidity-controlled incubator for $1 \mathrm{~h}$ before being given to the foster mother. During the resuscitation procedure, 20-min asphyxia pups presented gasping-like breathing, different color of the skin, and displayed less movements and vocalizations. After $1 \mathrm{~h}$ from asphyxia their appearance and behavioral reactivity were comparable to those of 0 -min pups. ANOVA evidenced a significant lower body weight in 20-min asphyxia pups [main effect of asphyxia: $F(2,42)=11.78 p=0.001 ; p$ $<0.01$ after post hoc comparisons]. Post hoc comparisons performed on the significant asphyxia $\times$ day interaction $[F(6$, $42)=2.42 p=0.095 ; p<0.05$ after post hoc comparisons] yielded a significant decrement in body weight gain in 20-min pups with respect to 0 -min group on pnd 5 and thereafter (Fig. 1). A main effect of sex was found $[F(1,42)=9.85 p=$ $0.016]$, with males weighing significantly more than females. 


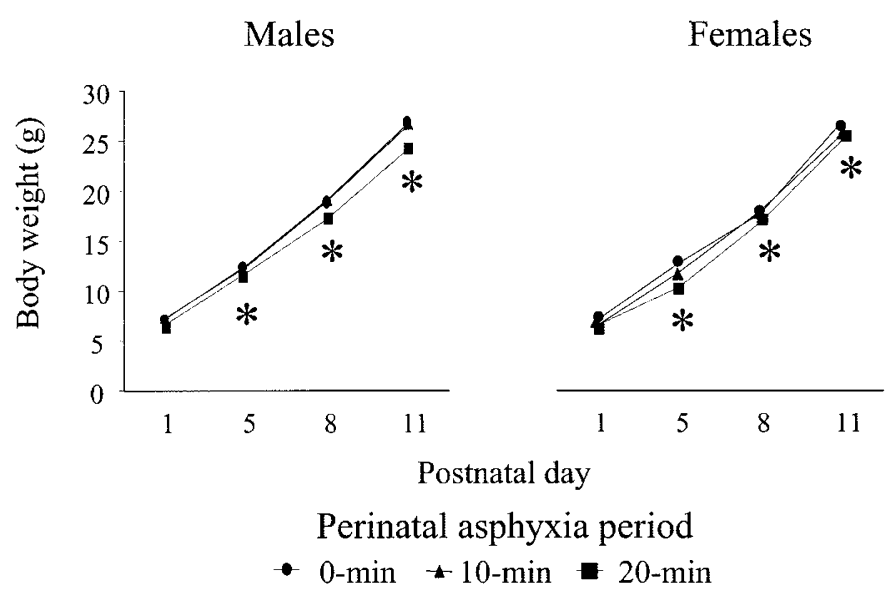

Figure 1. Body weight gain from pnd 1 to 11 of rats subjected at birth to different periods of perinatal asphyxia ( $n=8$ in each final group). *indicates a significant difference between the 0 -min and the 20-min group (asphyxia $\times$ day interaction). SEM $= \pm 0.27$.

Measurements of oxidative and inflammatory markers. Asphyxia affected 8-epi-PGF ${ }_{2 \alpha}$ levels $[F(2,42)=6,35 p<$ $0.003]$. The levels of 8 -epi- $\mathrm{PGF}_{2 \alpha}$ were significantly increased in 20-min asphyxia group $(p<0.01$ after post hoc comparisons), but not in animals subjected to shorter asphyctic episodes (Fig. 2A). We did not find sex differences in both control $(0.09 \pm 0.01 \mathrm{pg} / \mathrm{mg}$, females, $n=9 ; 0.11 \pm 0.02 \mathrm{pg} / \mathrm{mg}$, males, $n=8)$ and 20-min asphyctic $(0.24 \pm 0.07 \mathrm{pg} / \mathrm{mg}$, females, $n=7 ; 0.27 \pm 0.1 \mathrm{pg} / \mathrm{mg}$, males, $n=6$ ) pups. On the
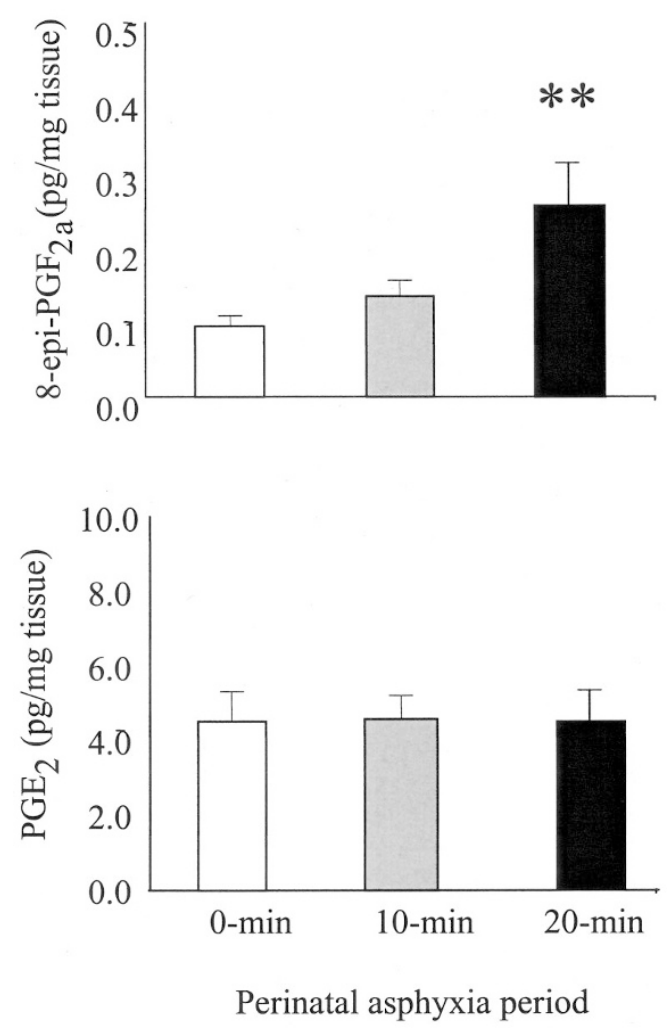

Figure 2. Brain levels of 8-epi- $\mathrm{PGF}_{2 \alpha}(A)$ and $\mathrm{PGE}_{2}(B)$ in control and asphyctic newborn rats $2 \mathrm{~h}$ after global ischemia. $* *$ indicates a significant difference between the 0 -min group and the 20-min group $(p<0.01)$. Male and female data are pooled. Data are means \pm SEM. contrary, the levels of $\mathrm{PGE}_{2}$, one of the major prostaglandin's synthesized during inflammatory processes, were not altered after 10 or $20 \mathrm{~min}$ of asphyxia when compared with control animals (Fig. 2B).

In addition, the inducible isoforms of $\mathrm{COX}(\mathrm{COX}-2)$ and iNOS were not detectable after the asphyctic episodes (Fig. 3). COX-2 expression was however detected in homogenates from adult rat brains. Cortex and hippocampus regions were chosen for their constitutive expression of COX-2, which begins after the first postnatal week (29).

Ultrasonic vocalization (pnds 5,8 , and 11). Statistical analysis was performed separately for pnd 5, 8, or 11 (Fig. 4). There was no effect of asphyxia on the total number of calls emitted by pups throughout the 3-min test. However, a significant change in the pattern of ultrasound emission was evident on pnd 11 [asphyxia $\times$ minute interaction: $F(4,32)=5.92$ $p=0.001]$. Post hoc comparisons evidenced a significant decrement of ultrasonic emission during the first minute in the 20 -min asphyxia group $(p<0.05)$ and then increase of vocalization. No significant interaction with sex was found. Finally, ANCOVA indicated that differences in ultrasound emission on pnd 11 were not related to the differences in body weight.

Spontaneous behavior (pnds 7, 12). Perinatal asphyxia affected spontaneous behavior on pnd 7 depending on sex and time block considered (Fig. 5). A lower duration of Twitching was found in 20-min asphyctic pups in the second time block (asphyxia $\times$ time block interaction: $F(4,36)=2.68 p=$ 0.0469; $p<0.05$ after post hoc comparisons). Furthermore, a lower frequency of Laying still events was shown by 20 -min asphyctic male pups in the first 5 min of the test [asphyxia $X$ sex $\times$ time-block interaction: $F(4,36)=2.64 p=0.049 ; p<$ 0.05 after post hoc comparisons]. Locomotion and Head moving did not appear to be significantly influenced by perinatal asphyxia. However, as a trend, 20-min asphyctic pups (both sexes) were more active in the first 5 min of the observation, [asphyxia $\times$ time block interaction: $F(4,36)=2.25 p=$ $0.082]$. In respect to controls, 20-min males displayed a different profile in Head moving duration, while an increase was found in 20-min female pups $[F(4,36)=2.32 p=0.075]$.

ANOVA carried out on data recorded on pnd 12 confirmed the alterations found on pnd 7 (see Fig. 6). Of notice, at this developmental stage some behavioral items are markedly sex-

\section{COX-2}

\section{iNOS}

\section{$\begin{array}{llllll}1 & 2 & 3 & 4 & \text { Cx } & \text { Hip }\end{array}$ \\ 0-min 20-min Adult}

Figure 3. Expression of COX-2 and iNOS in brain homogenates from control (lanes 1 and 2) and asphyctic (lanes 3 and 4) newborn rats $2 \mathrm{~h}$ after global ischemia by western blot analysis. Two animals representative of 5 are shown. Homogenates from cortex (Cx) and hippocampus (Hip) dissected from adult rat brain were analyzed as positive control for COX-2 expression. 


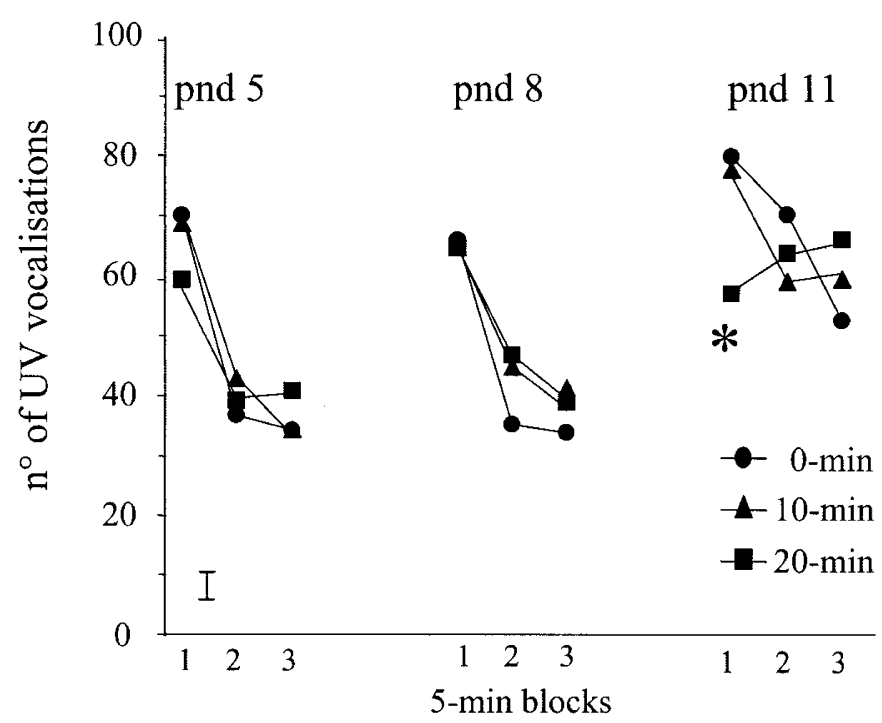

Figure 4. Number of ultrasounds emitted on pnds 5, 8, and 11 during a 3-min period by rats subjected at birth to different periods of perinatal asphyxia $(n=$ 18 in each final group, male and female data pooled). *indicates a significant difference between the 0 -min and the 20-min group. Vertical bar to the left indicates pooled SEM.

dimorphic (Locomotion, Head moving and Face washing). As a general trend, the effects of perinatal asphyxia seem to mirror these gender differences. Asphyxia significantly affected frequency and duration of Locomotion [asphyxia $\times$ sex interaction: $F(2,36)=7.04 p=0.005$, for frequency; $F(2,36)=2.89$ $p=0.081$, for duration], enhancing this behavior only in 20-min asphyctic females $(p<0.05$ after post hoc comparisons both for frequency and duration). Frequency and duration of Head moving were affected by perinatal asphyxia in a sexrelated fashion. Post hoc comparisons carried out on the significant asphyxia $\times$ sex interaction $[F(2,36)=4.98 p=0.018$; $F(2,36)=6.59 p=0.007$, respectively for frequency and duration], indicated reduction of this behavior in 20-min asphyctic males $(p<0.01)$. Conversely, Head moving was enhanced in 20-min asphyctic females $(p<0.01)$. As for Face washing frequency, a main effect of asphyxia $[F(2,36)=3.34$ $p=0.050]$ was found. Post hoc comparisons showed a significant reduction of this behavior only in the 10-min asphyxia group $(p<0.01)$. Finally, for Laying still duration, a significant asphyxia $\times$ sex interaction was found $[F(2,36)=5.74 p$ $=0.012]$, indicating enhanced hypoactivity in 20-min males $(p$ $<0.01$ after post hoc comparison). On the contrary, 20-min female pups spent less time in inactivity than the other groups ( $p<0.01$ after post hoc comparison).

Homing test (pnd 10). No significant differences among the different experimental conditions were observed in either homing response or locomotor activity. However, pups in the 20-min asphyxia group tended to take a longer time to reach the nest-scented area compared with the 0 - and 10-min groups, notwithstanding the number of crossings was comparable in the three groups (Table 1).

\section{DISCUSSION}

To our knowledge this is the first study attempting to relate an acute index of brain oxidative damage to behavioral sequels, monitored by analysis of early behavioral patterns, in a rat model of perinatal asphyxia. Our findings indicate that $20 \mathrm{~min}$ of global intrauterine asphyxia induces a very early increase in a specific marker of in vivo oxidative damage, such as the $\mathrm{F}_{2}$-isoprostane 8-epi- $\mathrm{PGF}_{2 \alpha}$, that appears to be predictive of delayed behavioral disturbances.

Oxidative stress, a condition in which free radical generation overwhelms anti-oxidant defenses, plays a role in the pathogenesis of several adult and infant brain diseases. $\mathrm{F}_{2}-$ isoprostanes are a class of PG-like compounds, formed by the direct attack of free radicals on arachidonic acid within cell membranes, and then released by phospholipase activities. Since their discovery (25), $\mathrm{F}_{2}$-isoprostanes have been proved to be specific and relatively stable markers of in vivo lipid peroxidation and their detection in brain tissues or CSF used to monitor the occurrence of oxidative stress in several adult brain pathologies (26). Recently, the levels of 8-epi- $\mathrm{PGF}_{2 \alpha}$, one of the major $\mathrm{F}_{2}$-isoprostanes formed in vivo, was found increased in the CSF of preterm infants with white matter damage (13), suggesting that this marker may be useful also in infant brain pathologies.

We found that brain levels of 8 -epi-PGF $2 \alpha$ were significantly increased in neonatal rats after 20-min global asphyxia. On the contrary $\mathrm{PGE}_{2}$, one of the major prostaglandin produced during inflammation, was not affected by asphyxia. Furthermore, there was no detectable induction of COX-2 and iNOS expression in early postasphyxia period; these two enzymes are responsible for the synthesis of PGs and nitric oxide, respectively, and have been recognized to play a role in adult brain hypoxic/ischemic damage (32). Although COX-2 expression has been found in brain sections from human infants, who died within 1-3 d after birth due to complication associated with prematurity or birth asphyxia (33) the lack of induction of COX-2, as well as iNOS, in our model is consistent with the short time elapsed between asphyxia and tissue sampling $(<2$ h) and suggests that lipid peroxidation (i.e. F2-isoprostane formation) occurs rapidly after hypoxia as a result of free radical production following re-perfusion and in the absence of an overt inflammatory response.

It is worth noting that COX-2 is normally expressed in selected neuronal populations of adult rat brain, particularly in the hippocampus and cortex, but it is detectable after the first postnatal week (34). This development-dependent expression of COX-2 is consistent with the absence of COX-2 in brain homogenates from neonatal rats reported in the present study.

As 8-epi-PGF ${ }_{2 \mathrm{a}}$ can exert biologic activities via membrane receptors for another arachidonic metabolite such as thromboxane $A_{2}(25)$, this lipid peroxidation could also be involved in the pathogenesis of brain damage after asphyxia. In this line, increased levels of 8-epi-PGF $2 \alpha$ have been proposed to contribute to placental vasoconstriction in preeclampsia (35).

On the behavioral side, we found that newborn rats experiencing 20-min asphyxia had reduced weight gain and show significant alterations in neonatal behavior pattern, such as ultrasound vocalization and spontaneous motor behavior.

In the rat species, ultrasounds are normally elicited after separation of the pup from the mother and siblings, and they have been described as a measure of the emotional distress 
MALES
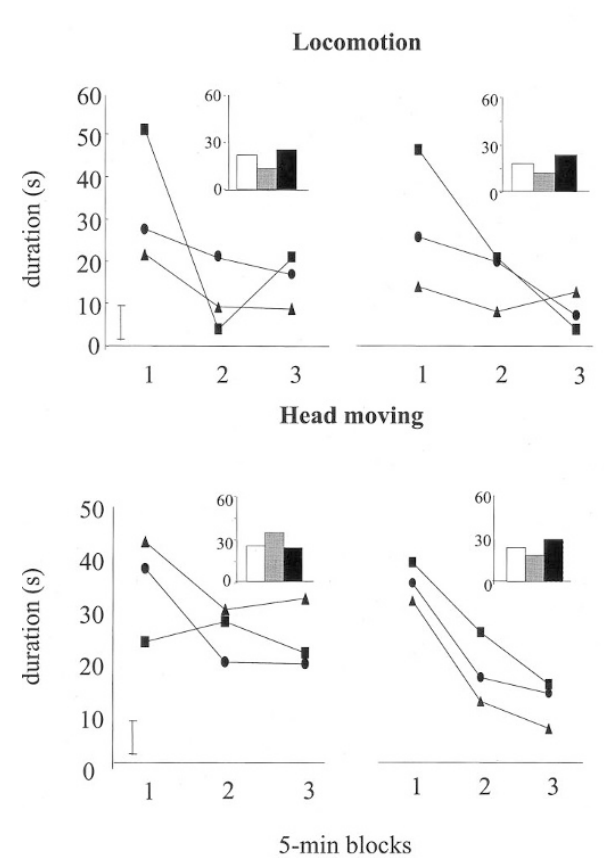

MALES

FEMALES

Twitching
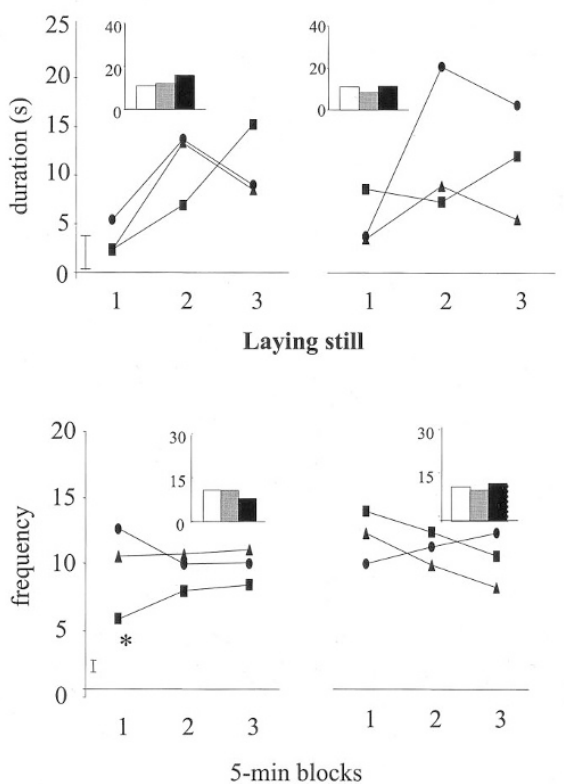

Perinatal asphyxia period

$\bullet \square 0$-min $₫ \square$ 10-min

20-min

Figure 5. Duration or frequency of different behavior items displayed by 7-d pups subjected at birth to different periods of asphyxia ( $n=10$ in each final group). *indicates a significant difference between the 0-min group and the 20-min group. For comparison with Fig. 6, mean values during the whole 15-min session are shown in bar plots. Vertical bars indicate pooled SEM.

\section{Locomotion}

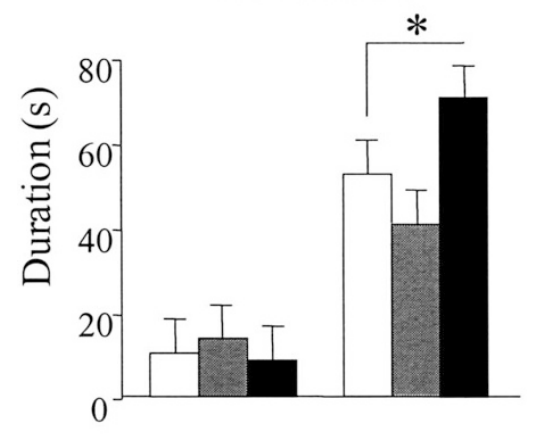

Laying still

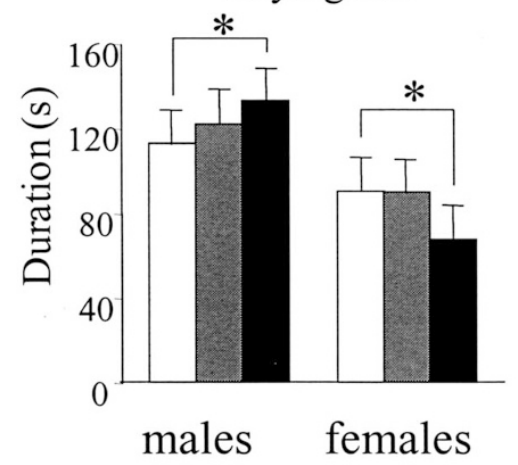

Head moving

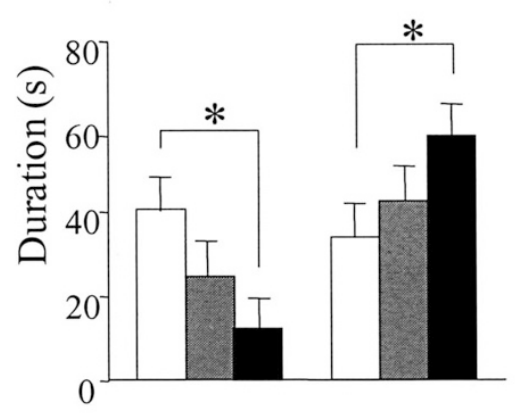

Face washing

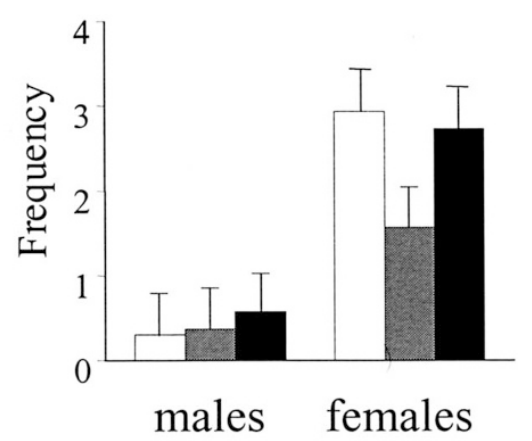

\section{0-min 0 10-min $\mathbf{\square} 20$-min}

\section{Perinatal asphyxia period}

Figure 6. Duration or frequency of different behavior items displayed by 12-d pups subjected at birth to different periods of asphyxia $(n=10$ in each final group). *indicates a significant difference between the 0 -min group and the 20-min group. Vertical bars are SEM. 
Table 1. Latency to reach the nest-scent area and number of crossings performed by 10-day rats in a 3-min test

\begin{tabular}{ccr}
\hline Asphyxia period & Crossings & Latency $(\mathrm{s})$ \\
\hline 0 -min & $6.3( \pm 0.8)$ & $55(12.0)$ \\
10 -min & $7.7( \pm 1.8)$ & $56(71.1)$ \\
20 -min & $7.4( \pm 1.4)$ & $138(66.0)$ \\
\hline
\end{tabular}

Data are: for crossings, mean $\pm \mathrm{SE}$; for latency, median and halfinterquartile range ((Q3-Q1)/2).

resulting from social isolation (36). Similarly, the ability to orient toward familiar olfactory cues is part of the adaptive response of the neonate rodent to isolation from the nest. Pups experiencing severe asphyxia tended to be slower in reaching the nest-scented area during the homing test, and displayed a different profile of ultrasound emission on day 11. Overall, these data suggest that global birth asphyxia might affect the maturation of those neurohormonal mechanisms involved in the adaptive response of the developing organisms to a stressor, such as maternal separation (37), in line with data reporting an altered response to stress in rats suffering perinatal hypoxia.

A clear effect of global asphyxia was found on spontaneous motor behavior, with an age dependent profile. On pnd 7 only some trends were observed, indicating a mild hyperactivity in 20-min asphyxia pups (both males and females) during the first $5 \mathrm{~min}$ of observation. Of notice, twitching behavior (an indicator of "active" sleep) is reduced in asphyctic pups in the central period of observation. Reduced twitching movements might again indicate that asphyctic pups are more aroused than control pups, in line with what suggested for the functional significance of this behavioral state (38). With the maturation of sensorimotor coordination, on pnd 12 , the effects on behavior became markedly significant. Such effects were sexually dimorphic and involved mainly forward locomotion and head movements. In particular, females tended to be hyperactive while males displayed hypoactivity. Hypoactivity has been previously reported in this model of perinatal global hypoxia in juvenile males (28 d) experiencing 20-min asphyxia, whereas 16-min asphyxia induced hyperactivity (15). As for sex differences, Loidl et al. (39) found that males subjected to 20-min asphyxia showed hypoactivity in an open-field at adulthood, while females were as active as controls. Whether the sexdimorphic effects of asphyxia on neonatal motor patterns reflect a different degree of severity of CNS damage it is difficult to say: however, the lack of any difference in 8 -epi-PGF ${ }_{2 \alpha}$ levels in males and females experiencing severe asphyxia suggests that gender-dependent factors might play a role in the delayed pathologic processes triggered by the asphyctic episode.

As for CNS alterations potentially underlying the early behavioral changes here reported, recent data indicated that most of the effects of global perinatal asphyxia appear around day 8. Van de Berg et al. (21) found marked apoptotic neuronal loss in the striatum peaking at pnd 8 in rats subjected to 20 -min asphyxia, supporting the view of delayed cell death. In line with such histopathological evidence, the alterations in ultrasound emission and spontaneous motor behavior did not appear before pnd 11, likely reflecting the delayed effect of the asphyctic insult on nerve cells. As a whole, the more significant effect of asphyxia is on spontaneous motricity. To date, the majority of data using this same perinatal asphyxia model concern the influence on motor behavior and on neurotransmitter systems related with overall activity, focusing on striatal dopaminergic system $(14,18,40)$. It is worth noting that qualitative alterations in spontaneous motricity have been also described in severely asphyxiated human infants (either preterm or term), and they are correlated with the severity of later neurologic outcome and with presence of brain lesions (11).

The present data do not allow drawing any direct causal link between $\mathrm{F}_{2}$-isoprostane increase and behavioral disturbances. However, the occurrence of behavioral changes only in pups belonging to the 20-min asphyxia condition, the same experimental condition inducing in pups sacrificed shortly after the insult $\mathrm{F}_{2}$-isoprostane alteration, suggest that brain 8-epi-PGF $2 \alpha$ levels might have a predictive value for delayed behavioral disturbances. Thus, this animal model might serve in the future as an evaluation tool of preventive and curative strategies to treat neurologic/behavioral disturbances associated with asphyxia. Furthermore, as du Plessis and Volpe (41) pointed out "... surrogate measures of brain injury are urgently needed within the first hours of life." In this context, these experimental findings might have some clinical relevance for the implementation of early diagnostic and prognostic strategies in the human newborn, provided that the increased $\mathrm{F}_{2}$-isoprostane levels can be measured in suitable human specimens such as CSF, blood, or urine.

\section{REFERENCES}

1. Vannucci RC 1990 Experimental biology of cerebral hypoxia-ischemia: relation to perinatal brain damage. Pediatr Res 27:317-326

2. Lorek A, Takei Y, Cady EB, Wyatt JS, Penrice J, Edwards AD, Peebles D, Wylezinska M, Owen-Reece H, Kirkbride V, Cooper CE, Aldridge RF, Roth SC, Brown G, Delpy DT, Reynolds EOR 1994 Delayed ("secondary") cerebral energy failure after acute hypoxia-ischemia in the newborn piglet: continuous 48 -h studies by phosphorus magnetic resonance spectroscopy. Pediatr Res 36:699-706

3. Northington FJ, Ferriero DM, Flock DL, Martin LJ 2001 Delayed neurodegeneration in neonatal rat thalamus after hypoxia-ischemia is apoptosis. J Neurosci 21:19311938

4. Vannucci RC, Perlman JM 1997 Interventions for perinatal hypoxic-ischemic encephalopathy. Pediatrics 100:1004-1014

5. Atkinson J, Anker S, Rae S, Weeks F, Braddick O, Rennie J 2002 Cortical visual evoked potentials in very low birthweight premature infants. Arch Dis Child Fetal Neonatal Ed 86:F28-31

6. Mercuri E, Ricci D, Cowan FM, Lessing D, Frisone MF, Haataja L, Counsell SJ, Dubowitz LM, Rutherford MA 2000 Head growth in infants with hypoxic-ischemic encephalopathy: correlation with neonatal magnetic resonance imaging. Pediatrics 106:235-243

7. Soul JS, Robertson RL, Tzika AA, du Plessis AJ, Volpe JJ 2001 Time course of changes in diffusion-weighted magnetic resonance imaging in a case of neonatal encephalopathy with defined onset and duration of hypoxic-ischemic insult. Pediatrics 108:1211-1214

8. Barkovich AJ, Westmark KD, Bedi HS, Partridge JC, Ferriero DM, Vigneron DB 2001 Proton spectroscopy and diffusion imaging on the first day of life after perinatal asphyxia: preliminary report. AJNR Am J Neuroradiol 22:1786-1794

9. Cioni G, Prechtl HF, Ferrari F, Paolicelli PB, Einspieler C, Roversi MF 1997 Which better predicts later outcome in full-term infants: quality of general movements or neurological examination? Early Hum Dev 50:71-85

10. Prechtl HF 1990 Qualitative changes of spontaneous movements in fetus and preterm infant are a marker of neurological dysfunction. Early Hum Dev 23:151-158

11. Prechtl HF, Einspieler C, Cioni G, Bos AF, Ferrari F, Sontheimer D 1997 An early marker for neurological deficits after perinatal brain lesions. Lancet 349:1361-1363

12. Nagdyman N, Komen W, Ko HK, Muller C, Obladen M 2001 Early biochemical indicators of hypoxic-ischemic encephalopathy after birth asphyxia. Pediatr Res 49:502-506

13. Inder T, Mocatta T, Darlow B, Spencer C, Volpe JJ, Winterbourn C 2002 Elevated free radical products in the cerebrospinal fluid of VLBW infants with cerebral white matter injury. Pediatr Res 52:213-218 
14. Bjelke B, Andersson K, Ogren SO, Bolme P 1991 Asphyctic lesion: proliferation of tyrosine hydroxylase-immunoreactive nerve cell bodies in the rat substantia nigra and functional changes in dopamine neurotransmission. Brain Res 543:1-9

15. Romijn HJ, Hofman MA, Gramsbergen A 1991 At what age is the developing cerebral cortex of the rat comparable to that of the full-term newborn human baby? Early Hum Dev 26:61-67

16. Boksa P, Zhang Y, Bestawros A 2002 Dopamine d1 receptor changes due to caesarean section birth: effects of anesthesia, developmental time course, and functional consequences. Exp Neurol 175:388-397

17. Boksa P, Krishnamurthy A, Brooks W 1995 Effects of a period of asphyxia during birth on spatial learning in the rat. Pediatr Res 37:489-496

18. Chen Y, Hillefors-Berglund M, Herrera-Marschitz M, Bjelke B, Gross J, Andersson K, von Euler G 1997 Perinatal asphyxia induces long-term changes in dopamine D1, D2, and D3 receptor binding in the rat brain. Exp Neurol 146:74-80

19. Chen Y, Herrera-Marschitz M, Bjelke B, Blum M, Gross J, Andersson K 1997 Perinatal asphyxia-induced changes in rat brain tyrosine hydroxylase-immunoreactive cell body number: effects of nicotine treatment. Neurosci Lett 221:77-80

20. Dell'Anna E, Chen Y, Engidawork E, Andersson K, Lubec G, Luthman J, HerreraMarschitz M 1997 Delayed neuronal death following perinatal asphyxia in rat. Exp Brain Res 115:105-115

21. Van de Berg WD, Schmitz C, Steinbusch HW, Blanco CE 2002 Perinatal asphyxia induced neuronal loss by apoptosis in the neonatal rat striatum: a combined TUNEL and stereological study. Exp Neurol 174:29-36

22. El-Khodor BF, Boksa P 1998 Birth insult increases amphetamine-induced behavioral responses in the adult rat. Neuroscience 87:893-904

23. Kohlhauser C, Kaehler S, Mosgoeller W, Singewald N, Kouvelas D, Prast H, Hoege H, Lubec B 1999 Histological changes and neurotransmitter levels three months following perinatal asphyxia in the rat. Life Sci 64:2109-2124

24. Taylor DL, Edwards AD, Mehmet H 1999 Oxidative metabolism, apoptosis and perinatal brain injury. Brain Pathol 9:93-117

25. Lawson JA, Rokach J, FitzGerald GA 1999 Isoprostanes: formation, analysis and use as indices of lipid peroxidation in vivo. J Biol Chem 274:24441-24444

26. Greco A, Minghetti L, Levi G 2000 Isoprostanes, novel markers of oxidative injury, help understanding the pathogenesis of neurodegenerative diseases Neurochem Res 25:1357-1364

27. Enters EK, Spear LP 1988 Serotonergic and opiate interactions in the modulation of drug- and environmental-induced analgesia in the neonatal rat pup. Behav Neural Biol 50:80-97
28. Santucci D, Cagiano R, Calamandrei G 1994 IGF-I and IGF-I24-41 but not IGF-I57-70 affect somatic and neurobehavioral development of newborn male mice. Brain Res Bull 35:367-371

29. Venerosi A, Valanzano A, Alleva E, Calamandrei G 2001 Prenatal exposure to anti-HIV drugs: neurobehavioral effects of zidovudine (AZT) + lamivudine (3TC) treatment in mice. Teratology 63:26-37

30. Zorrilla EP 1997 Multiparous species present problems (and possibilities) to developmentalists. Dev Psychobiol 30:141-150

31. Wilcox R 1987 New statistical procedures for the social sciences. Modern solutions to basic problems. Lawrence Erlbaum, Hillsdale, NJ, pp 182-187

32. Iadecola C, Alexander M 2001 Cerebral ischemia and inflammation. Curr Opin Neurol 14:89-94

33. Toti P, De Felice C, Schurfeld K, Stumpo M, Bartolommei S, Lombardi A, Petraglia E, Buonocore G 2001 Cyclooxygenase-2 immunoreactivity in the ischemic neonatal human brain. An autopsy study. J Submicrosc Cytol Pathol 33:245-249

34. Kaufmann WE, Worley PF, Pegg J, Bremer M, Isakson P 1996 COX-2, a synaptically induced enzyme, is expressed by excitatory neurons at postsynaptic sites in rat cerebral cortex. Proc Natl Acad Sci USA 93:2317-2321

35. Walsh SW, Vaughan JE, Wang Y, Roberts LJ, 2nd 2000 Placental isoprostane is significantly increased in preeclampsia. FASEB J 14:1289-1296

36. Hofer MA, Shair H 1978 Ultrasonic vocalization during social interaction and isolation in 2-weeek-old rats. Dev Psychobiol 11:495-504

37. Rosenfeld P, Wetmore JB, Levine S 1992 Effects of repeated maternal separations on the adrenocortical response to stress of preweanling rats. Physiol Behav 52:787-791

38. Blumberg MS, Stolba MA 1996 Thermogenesis, myoclonic twitching, and ultrasonic vocalization in neonatal rats during moderate and extreme cold exposure. Behav Neurosci 110:305-314

39. Loidl CF, Gavilanes AW, Van Dijk EH, Vreuls W, Blokland A, Vles JS, Steinbusch HW, Blanco CE 2000 Effects of hypothermia and gender on survival and behavior after perinatal asphyxia in rats. Physiol Behav 68:263-269

40. Chen Y, Ogren SO, Bjelke B, Bolme P, Eneroth P, Gross J, Loidl F, HerreraMarschitz M, Andersson K 1995 Nicotine treatment counteracts perinatal asphyxiainduced changes in the mesostriatal/limbic dopamine systems and in motor behaviour in the four-week-old male rat. Neuroscience 68:531-538

41. du Plessis AJ, Volpe JJ 2002 Perinatal brain injury in the preterm and term newborn. Curr Opin Neurol 15:151-157 